\title{
Intramuscular midazolam versus intravenous lorazepam for the prehospital treatment of status epilepticus in the pediatric population
}

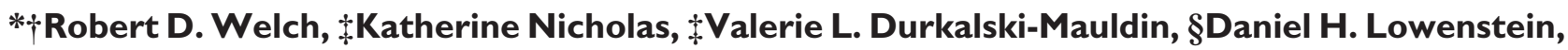 \\ đRobin Conwit, **Prashant V. Mahajan, $\dagger+$ Christopher Lewandowski, + + Robert Silbergleit, and \\ For the Neurological Emergencies Treatment Trials (NETT) Network Investigators'
}

\author{
Epilepsia, 56(2):254-262, 2015 \\ doi: 10.1111/epi.12905
}

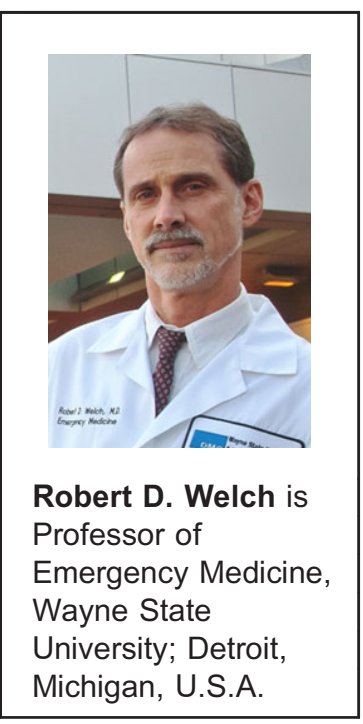

\begin{abstract}
SUMMARY
Objective: To examine the effectiveness of intramuscular (IM) midazolam versus intravenous (IV) lorazepam for the treatment of pediatric patients with status epilepticus (SE) in the prehospital care setting.

Methods: This multicenter clinical trial randomized patients diagnosed with SE to receive either IM midazolam or IV lorazepam administered by paramedics in the prehospital care setting. Included in this secondary analysis were only patients younger than 18 years of age. Evaluated were the associations of the treatment group (IM vs. IV) with the primary outcome, defined as seizure cessation prior to emergency department (ED) arrival, and with patient characteristics, time to important events, and adverse events. Descriptive statistics and $\mathbf{9 9 \%}$ confidence intervals $(\mathrm{Cls})$ were used for the analysis.

Results: Of 893 primary study subjects, 120 met criteria for this study ( 60 in each treatment group). There were no differences in important baseline characteristics or seizure etiologies between groups. The primary outcome was met in $41(68.3 \%)$ and 43 (7I.7\%) of subjects in the IM and IV groups, respectively (risk difference [RD] $-3.3 \%$, 99\% $\mathrm{Cl}-24.9 \%$ to $18.2 \%$ ). Similar results were noted for those younger than I I years (RD $-1.3 \%, 99 \% \mathrm{Cl}-25.7 \%$ to $23.1 \%$ ). Time from initiating the treatment protocol was shorter for children who received IM midazolam, mainly due to the shorter time to administer the active treatment. Safety profiles were similar.

Significance: IM midazolam can be rapidly administered and appears to be safe and effective for the management of children with SE treated in the prehospital setting. The results must be interpreted in the context of the secondary analysis design and sample size of the study.
\end{abstract}

KEY WORDS: Status epilepticus, Prehospital treatment, Pediatrics.

Accepted November 29, 2014; Early View publication January 17, 2015.

*Department of Emergency Medicine, Wayne State University School of Medicine, Detroit, Michigan, U.S.A.; $\dagger$ Cardiovascular Research Institute, Wayne State University School of Medicine, Detroit, Michigan, U.S.A.; \$Department of Public Health Sciences, Medical University of South Carolina, Charleston, South Carolina, U.S.A.; §Department of Neurology, University of California, San Francisco, San Francisco, California, U.S.A.; 9 The National Institute of Neurological Disorders and Stroke, National Institutes of Health, Bethesda, Maryland, U.S.A.; **Department of Pediatrics, Wayne State University School of Medicine, Detroit, Michigan, U.S.A.; ††Department of Emergency Medicine, Henry Ford Health System, Detroit, Michigan, U.S.A.; and \$\$Department of Emergency Medicine, University of Michigan, Ann Arbor, Michigan, U.S.A.

${ }^{1}$ See list of NETT Network Investigators in Appendix 1.

Address correspondence to Robert D. Welch, Department of Emergency Medicine, Wayne State University School of Medicine, Detroit Receiving Hospital - 6G/UHC, 4201 St. Antoine, Detroit, MI, 48201 U.S.A. E-mail: rwelch@med.wayne.edu

Wiley Periodicals, Inc.

(C) 2015 International League Against Epilepsy 
Although seizures arise from many etiologies, prolonged seizure activity from any cause is a dangerous neurologic emergency in both adults and children. Regardless of the initially precipitating cause, seizures that do not terminate spontaneously or with early treatment often create positive feedback loops in which the seizure becomes self-perpetuating, resulting in status epilepticus (SE). Despite its many disparate triggers, SE is a relatively homogenous pathophysiologic process for which the initial acute treatment, benzodiazepines, is the same regardless of the etiology or age of the patient. ${ }^{1,2}$ In the prehospital setting and in the emergency department (ED), conventional first-line therapy for patients with SE is intravenous (IV) diazepam or lorazepam. ${ }^{2,3}$ However, establishing an IV line in convulsing patients, especially in the prehospital setting, can be challenging and can delay treatment. Intramuscular (IM) delivery of midazolam has been advocated as an alternative treatment, but prior to 2012, it had not been demonstrated to be as safe or effective as IV treatment. ${ }^{4,5}$

The Rapid Anticonvulsant Medication Prior to Arrival Trial (RAMPART) was a randomized controlled clinical trial designed to determine whether IM midazolam was noninferior to IV lorazepam in adults and children with prehospital SE. ${ }^{6}$ Published in 2012, RAMPART showed that those randomized to IM midazolam were more likely to have terminated seizures prior to ED arrival and were less likely to require hospital ward or intensive care unit (ICU) admission. RAMPART included pediatric patients because children and adolescents constitute a substantial proportion of those with prehospital SE. Preliminary surveys were performed to ensure that the study population would have a sufficient representation of children. Given the consistency of the underlying biology of SE in adults and children, RAMPART was powered to test the primary noninferiority hypothesis in the overall cohort with results applicable to the general population of patients. Although it was not designed to evaluate efficacy separately in either the adult or pediatric subpopulation, the data obtained included a large number of nonadult patients.

The RAMPART study represents the largest prospective clinical trial experience with prehospital SE in children and adolescents. The goal of this study is to describe and clinically characterize this unique cohort. Post hoc subgroup analyses by age and safety outcomes by age are also explored.

\section{Methods}

\section{Study design and setting}

This study is a secondary analysis of the RAMPART clinical trial database focusing on patients younger than 18 years of age. The setting was prehospital and included 33 emergency medical services (EMS) agencies in the United States and involved 4,314 paramedics trained to enroll patients. A total of 79 hospitals were able to receive enrolled patients. RAMPART was a prospective intentionto-treat noninferiority trial conducted under exception from informed consent (EFIC) for emergency research (21 Code of Federal Regulations 50.24). Institutional review board approval was obtained locally by all participating sites. Details of the study have been reported previously and are available elsewhere. ${ }^{6,7}$

\section{Selection of participants}

Participants were enrolled in RAMPART in the prehospital setting after being evaluated by paramedics and found to be in SE. Participants were adults of any size or children with an estimated body weight of $\geq 13 \mathrm{~kg}$. Estimated weight was determined by an adaption of a length-based weight estimation tool. ${ }^{8}$ More details of the tool's development for this study are available through the online protocol (see Table 5). ${ }^{9}$ The trial used the contemporary operational definition of SE; seizure activity persisting for $>5$ min or lack of return to baseline function between seizures within a 5 min interval. ${ }^{10}$ Excluded were those in which the seizures required alternate treatments (major trauma, hypoglycemia, cardiac arrest, heart rate of $<40$ beats per minute) or patients with a known allergy to study medication. Additional exclusions were patients who opted out (defined as wearing a bracelet marked "RAMPART declined"), prisoners, known pregnancy, or known enrollment in another clinical study.

For the current analysis, the group of interest was the RAMPART pediatric population, defined as all participants younger than 18 years of age. To further examine for potential age-group-based differences, the pediatric patient study population was also subdivided into the following age groups; 0-5, 6-10, and 11-17 years of age. These age groupings were utilized for the following reasons: (1) the febrile seizure range is usually up to 5 years of age and this is the "preschool" age, and (2) using narrower age ranges for groups would result in very small numbers in each age group. We also examined the primary outcome result for the group of "children" defined as younger than 11 years of age. Because febrile seizures are unique to pediatric patients, we examined certain features of patients with that etiology. Sensitivity analyses were performed to determine whether any part of the analysis was markedly altered by small changes to these thresholds.

\section{Interventions}

All participants in RAMPART received active treatment with either IM midazolam or IV lorazepam at one of two doses. Study intervention was initiated by opening an instrumented study box containing a study drug kit. Each kit contained two color-coded shrink-wrapped study drug bundles, one for each dose tier, consisting of one IM autoinjector (Investigational Midazolam Autoinjector, Meridian Medical Technologies, Columbia, MD, U.S.A.) and one prefilled IV syringe (Carpuject System, Hospira, Lake Forest, 
IL, U.S.A.). All adults and those children with an estimated weight of more than $40 \mathrm{~kg}$ received $10 \mathrm{mg}$ midazolam IM followed by IV placebo or IM placebo followed by $4 \mathrm{mg}$ lorazepam IV. In children with estimated weights of 13$40 \mathrm{~kg}$, the active treatment was $5 \mathrm{mg}$ midazolam IM or $2 \mathrm{mg}$ lorazepam IV. Blinding and simple randomization with equal allocation were achieved using a double dummy strategy in which each kit was randomly assigned either placebo-IM-medication/active-IV-medication or active-IMmedication/placebo-IV-medication at the central pharmacy. All subjects were treated with the IM autoinjector, followed immediately by obtaining venous access and treatment with the IV syringe. If IV access could not be obtained, intraosseous (IO) access was permitted and considered to be a vascular access route equivalent to IV access. In the event that the seizure activity continued for $>10 \mathrm{~min}$, rescue medication therapy, based on local EMS protocol, was administered.

\section{Methods and measurements}

Study measurements in the prehospital phase were undertaken utilizing a voice-recorded and time-stamped data logger that was integrated into the study box. The voice and time recorder was automatically activated when the study box was opened. Data points collected by EMS personnel included time IM medication given, time IV access obtained, time IV medication given, time convulsions stopped if applicable, and whether the patient was still convulsing at the time of hospital arrival. Paramedics were trained to explicitly verbalize these events when they occurred, which were captured by the voice recorder. Time stamps were abstracted from the recordings at the study clinical coordinating center.

Once the patient arrived in the ED, trained study coordinators or study physicians queried the treating ED physician to determine if the patient was still thought to be seizing upon arrival at the receiving ED (the primary outcome). Other measurements, including patient demographics, medical history, need for endotracheal intubation, hospitalization, ICU care, and length of stay were collected prospectively in the ED, and if hospitalized, during the inpatient stay and at the time of hospital discharge.

\section{Outcomes}

The primary outcome was termination of the seizure prior to ED arrival as determined by the attending ED physician. Secondary outcomes included the time intervals from box open to termination of convulsions, from box open to active drug administration, and from active drug administration to termination of convulsions. We also examined a number of other clinical parameters and events such as seizure etiology, need for rescue medication, need for hospitalization or ICU care, endotracheal intubation, recurrent seizure within $12 \mathrm{~h}$ of ED arrival, and mortality.

\section{Analysis}

Baseline patient characterizes and hospital events were reported using number of events and proportions for categorical outcomes with associated $99 \%$ confidence intervals (CIs) for binomial proportions or means and standard deviations when describing the entire pediatric study population characteristics. Between-group data were analyzed using descriptive statistics to characterize baseline demographics and clinical outcome variables. For differences between treatment groups, categorical variables were compared by differences in proportions with associated $99 \%$ CIs, and for continuous data we reported differences in means and the associated $99 \%$ CIs. Because this was a secondary analysis, no a priori sample size calculation was performed and a noninferiority analysis was not utilized for the primary outcome. It must be emphasized that $99 \%$ CIs were used because of the number of comparisons and features evaluated; this choice resulted in wider (and more conservative) confidence intervals than the more traditional 95\% CI.

Finally, formal statistical testing to generate p-values was not done for a variety of reasons: subjects were not randomized by age strata, it was a secondary analysis, and study sample size was not sufficient to control for potential type 2 errors. Data analyses were performed using SAS version 9.2 or higher (SAS, Cary, NC, U.S.A.).

\section{RESULTS}

\section{Clinical characteristics}

The RAMPART pediatric study population consisted of 120 patients between the ages of 0 and 17 years of age (Fig. 1), with the highest prevalence of enrollments in preschool-age children. Pediatric patients accounted for $13.4 \%$ of 893 patients in the entire RAMPART study. The mean age was 6.7 years (99\% CI 5.6-7.8). Figure 2 shows the overall age distribution. For the age subgroups defined in the methods, 61 were $0-5$ years, 35 were $6-10$ years and, 24 were 11-17 years of age. Two patients enrolled (ages 9 months and 1 year old) that violated protocol as a result of misapplication of the length-based weight estimation tool were not included in the analysis.

Race and ethnicity are shown in the Table 1 . These generally reflected the demographics of the communities in which the study was conducted. Race and ethnicity in the pediatric cohort differed slightly from that of the overall study cohort (data not shown). This is likely the effect of a limited number of communities in which children were not enrolled for some part or the entirety of the study's duration.

About one third of the subjects had a new-onset seizure, whereas the remainder had a prior history of seizure. Fever was considered to be the seizure etiology in 23 of 105 subjects $(21.9 \%$; $99 \%$ CI $11.5-32.3 \%)$ with a known etiology (10 in the IV group and 13 in the IM group). Medication noncompliance was a relatively infrequent cause of seizures 


\section{Figure I.}

Flow chart describing the selection of the pediatric study population (for more details see the RAMPART study report). ${ }^{6}$

Epilepsia (C) ILAE
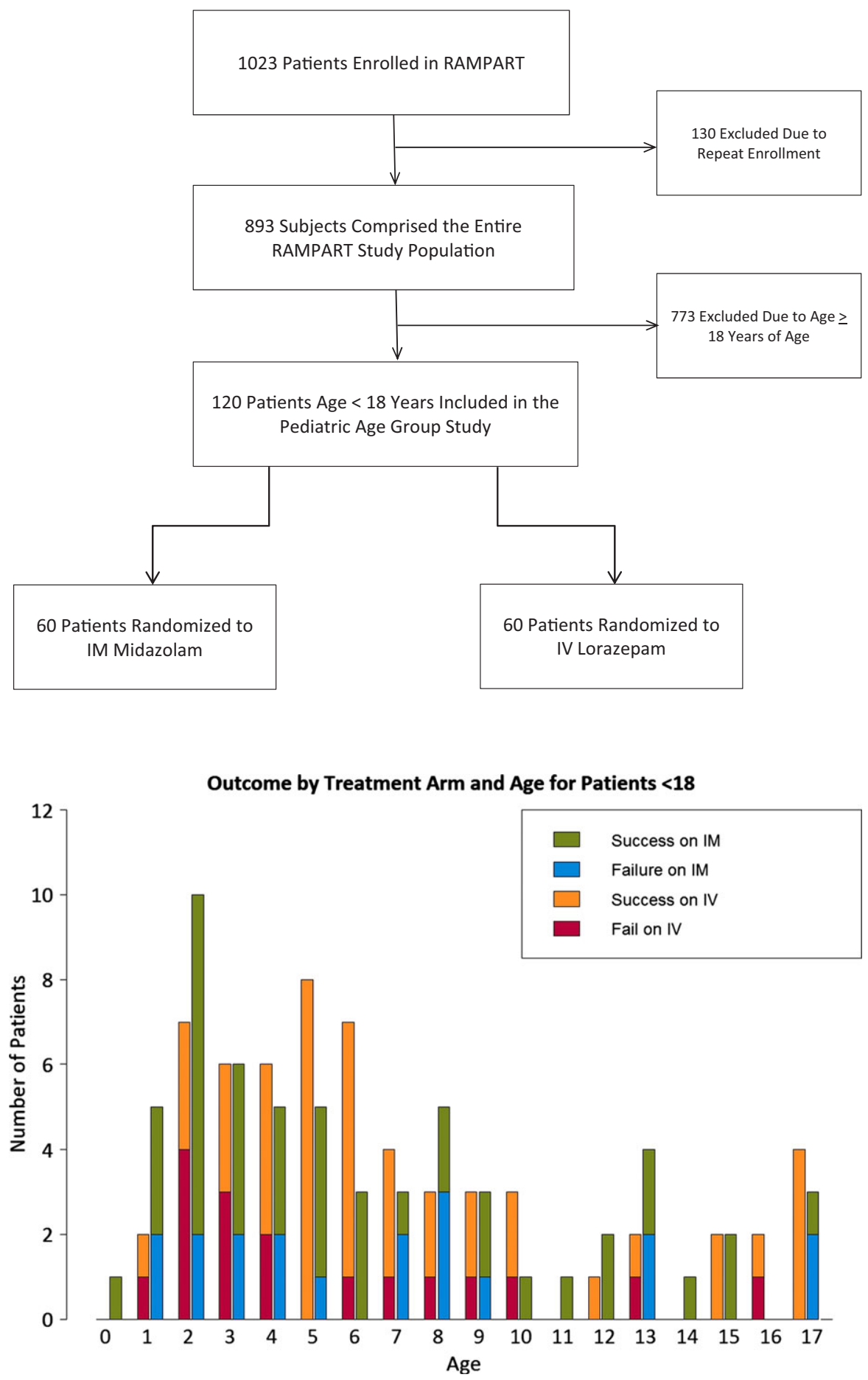

\section{Figure 2.}

Distribution and treatment outcomes of the study popluation by year of age; IV, intravenous lorazepam; IM, intramuscular midazolam. Epilepsia (C) ILAE $(\mathrm{n}=12$ of 105 patients; $11.4 \%$, 99\% CI 3.4-19.4\%). Ten percent of pediatric patients were ultimately determined to have nonepileptic spells, and the majority of these were psychogenic seizures.

Rescue medication was required in the prehospital setting in only $11.7 \%$ (99\% CI $4.1-19.2 \%$ ) of all study patients. There were no deaths among the 120 pediatric patients. Among the 120 pediatric subjects, 57 were hospitalized, with 21 of these admitted to the ICU. Thirteen subjects overall were intubated.

There were 23 patients with a final diagnosis in the medical record of febrile seizures. Two of these patients were 6 years of age and one was 8 years of age (it was unclear why these patients who were older than 5 years of age were diagnosed with febrile seizures). For the patients with febrile seizures, 10 were hospitalized, of 


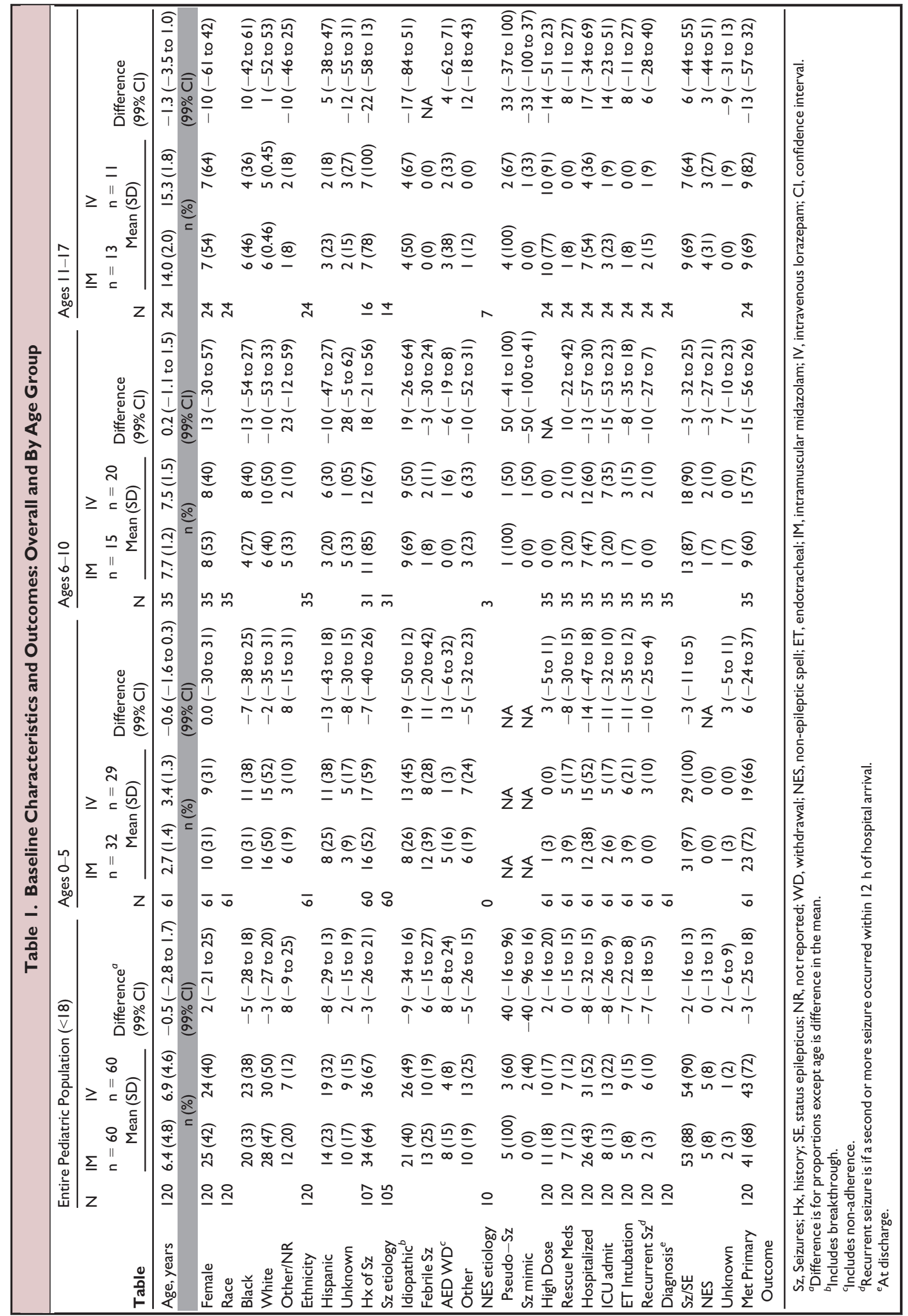


whom 4 went to the ICU, 2 required endotracheal intubation, and 14 met the primary outcome of seizure cessation prior to hospital arrival. The table details the overall study patient population characteristics and those same characteristics by age group (Table 1).

\section{Primary outcome}

In the overall pediatric group, 60 patients were randomized to the IM midazolam group and 60 to the IV lorazepam group. There were no major differences in patient demographics, the cause of status epilepticus, or the proportion of patients receiving each dose tier.

For the primary outcome of seizure cessation prior to ED arrival, $41(68.3 \%)$ of 60 patients who received treatment with IM midazolam met the primary outcome, as compared to $43(71.6 \%)$ of 60 treated with IV lorazepam (risk difference $=-3.3 \% ; 99 \% \mathrm{CI}-24.9 \%$ to $18.2 \%$ ). In addition, for children younger than 11 years of age, $32(68.1 \%)$ of 47 and $34(69.4 \%)$ of 49 patients in the IM and IV groups, respectively, met the primary outcome (risk difference $=-1.3 \%$, $99 \%$ CI $-25.7 \%$ to $23.1 \%$ ).

\section{Secondary outcomes}

Point estimates of the secondary outcome measures all favored IM midazolam. Fewer patients in the IM midazolam group who were hospitalized had recurrent seizures, were intubated, or required ICU care. The mean length-of-stay (excluding those who were not admitted rather than considering them as zero day lengths of stay) was 2.6 days in the IM group compared to 2.0 days in the IV group, but median lengths of stay were similar.

When stratified by the defined age subgroups, similar results were noted for patient characteristics, seizure etiology and characteristics, in-hospital events, and the primary outcome. The point estimates for treatment differences favored the IV group for the two older age strata (IM therapy was favored for those younger than 6 years of age), but again the number of patients per group is very small and caution must be used when evaluating these subgroup results (see the Table 1).

\section{Time interval outcomes}

Figure 3A shows the distribution of times from study box opening to treatment, study box opening to seizure termination, and from treatment to seizure termination. As a comparison, Figure 3B shows the same data for the adult ( $\geq 18$ years old) RAMPART study patients. In both groups, the same trends can be seen - particularly time from study box opening to seizure termination appears to be faster for patients treated with IM midazolam (no formal statistical testing was done). Not surprisingly, it was noted that time to initiation of active treatment may be shorter for those receiving IM midazolam as compared to IV lorazepam, but the time from active treatment to seizure termination was shorter in the IV lorazepam group. The same times to events
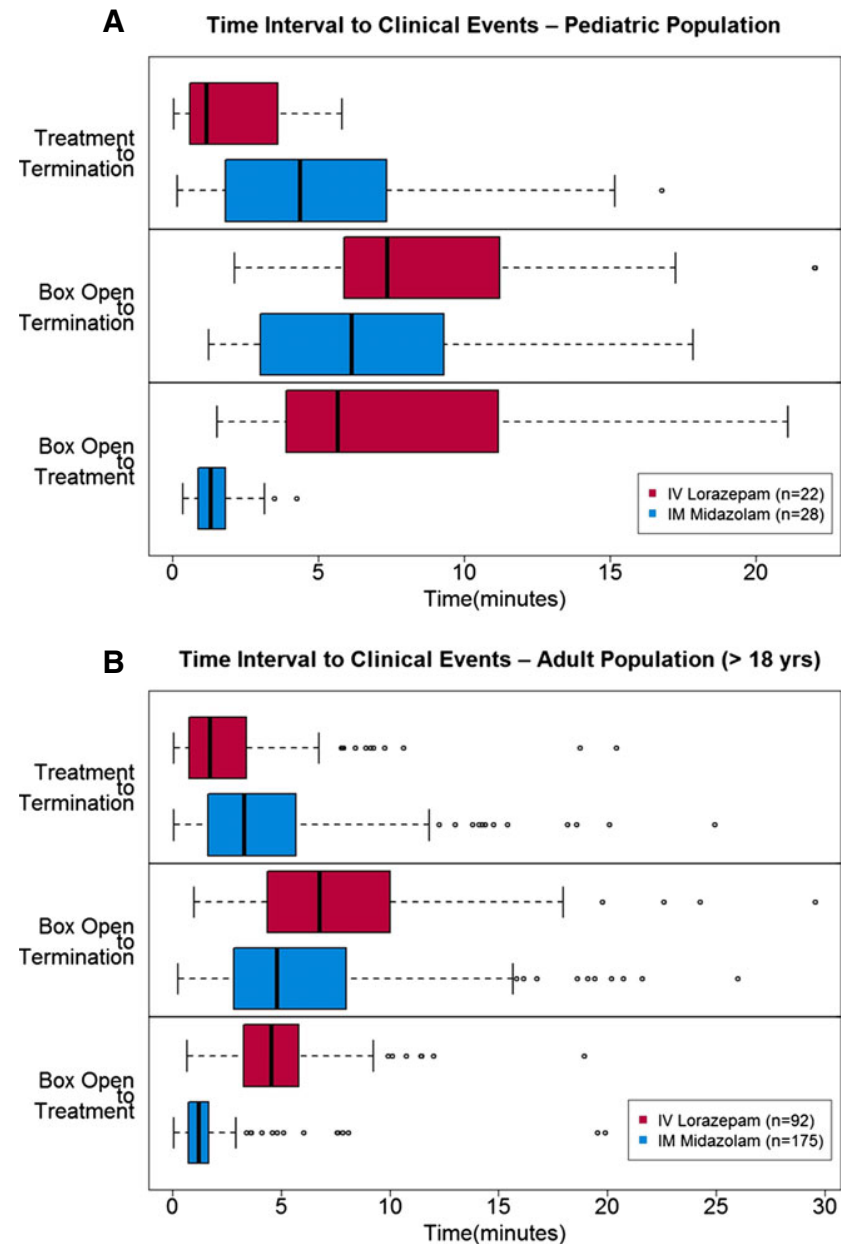

Figure 3.

(A, B) Time intervals to predefined clinical treatment and outcome events (as described on the figures; note the time intervals on the $x$-axis are not the same for each figure due to scale and outliers). Boxes are the interquartile ranges, bold vertical lines within boxes are median times, whisker bars are 1.5 times the interquartile range, and circles represent outliers.

as recorded by the data logger also showed similar trends among the three age strata (Fig. 4A, B, and C).

Median time intervals from first treatment encounter (defined as the IM dose; either active drug or placebo) to hospital ED arrival were $16.5 \mathrm{~min}$ (13.8 to $25.1 \mathrm{~min} ; 25^{\text {th }}$ to 75th percentiles) for the IM group and $19.6 \mathrm{~min}$ (15.0 to $27.1 \mathrm{~min}$ ) for the IV group.

\section{Discussion}

The RAMPART study clearly demonstrated that IM midazolam is a safe and effective alternative therapy for the treatment of SE when used by paramedics in the prehospital setting in a population inclusive of both adult and pediatric patients. It also highlighted findings consistent with underlying assumptions that permitted including 
A Time Interval to Clinical Events - Pediatric Population (0 - 5 yrs)

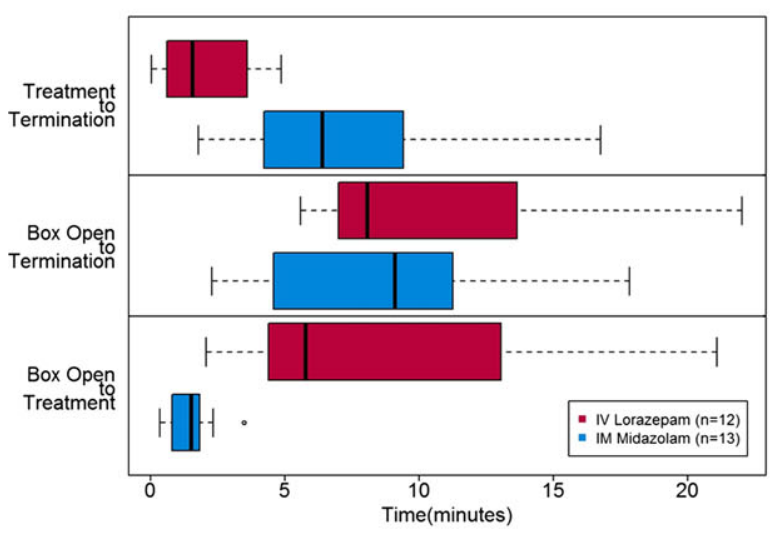

B Time Interval to Clinical Events - Pediatric Population (6 - 10 yrs)

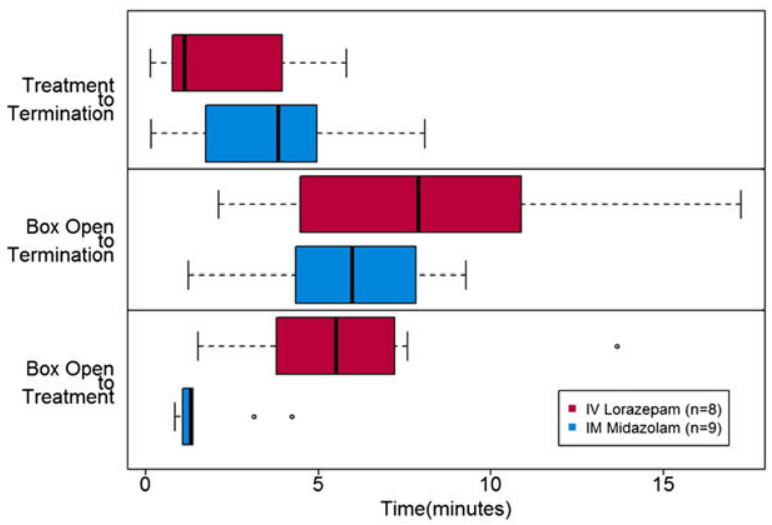

C Time Interval to Clinical Events - Pediatric Population (11 - 17 yrs)

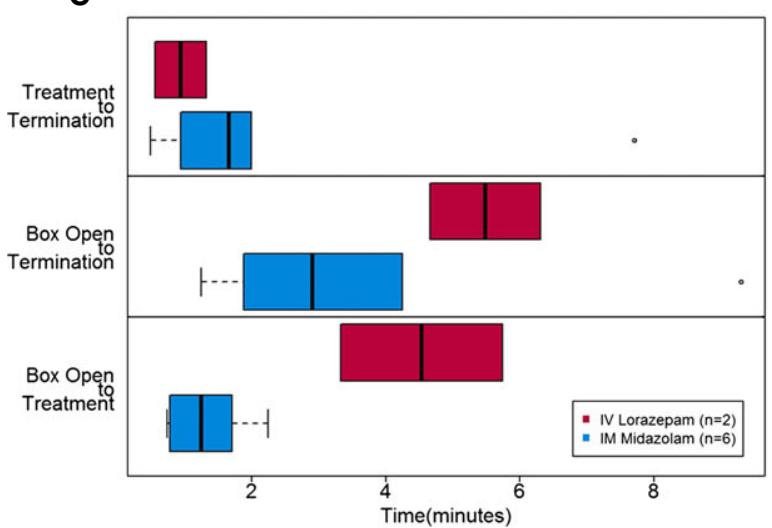

Figure 4.

(A, B, C) Time intervals to predefined clinical treatment and outcome events (as described on the figures; note the time intervals on the $\mathrm{x}$-axis are not the same for each figure due to scale and outliers). Boxes are the interquartile ranges, bold vertical lines within boxes are median times, whisker bars are 1.5 times the interquartile range, and circles represent outliers.

Epilepsia (c) ILAE

adults and children in the parent trial. Furthermore, this secondary analysis of the RAMPART pediatric cohort implies there is likely an acceptable safety profile within reasonable margins of confidence. A survey of experts found they clearly favor lorazepam as the first-line treatment of choice for SE in general, and guidelines suggest that lorazepam is the preferred agent when IV therapy is utilized. ${ }^{2,11}$ The recent Pediatric Seizure Study, however, found that IV lorazepam offered no benefit over IV diazepam for treating children with SE in the ED, and therefore questions the concept of lorazepam superiority, at least in children. ${ }^{12}$ Because many EMS systems have adopted IM midazolam as a treatment for SE, the findings add important observations needed to continue treatment protocols and policies for pediatric patients.

Prior studies have demonstrated that midazolam and nonIV routes of rapid-acting benzodiazepine anticonvulsant medications are effective and desirable. A meta-analysis of midazolam versus diazepam showed that non-IV midazolam was safe and effective in children and adults. ${ }^{13}$ One study found nasal midazolam to be effective for children with prolonged febrile seizures treated in the ED, and another found overwhelming preference by caregivers in Europe for using nasal or buccal midazolam for seizures. ${ }^{14,15}$ Our study adds to prior work in finding the IM route to be a reasonable alternative to IV medication when treating SE in the prehospital setting. Anecdotally, we noted preference for this route of administration among parents, school nurses, and other caretakers when performing the community consultation required for EFIC.

It is also interesting that a relatively low proportion of cases of SE was attributed to medication noncompliance among pediatric patients (11.4\%; 99\% CI 3.4-19.4\%) compared to $38.5 \%$ (99\% CI $33.7-43.3 \%$ ) for those older than 17 years of age and $34.9 \%$ (99\% CI 30.6-39.3\%) in the overall RAMPART study population. It remains to be determined whether this has age-derived treatment implications once the patient arrives in the ED, such as immediate administration of a large dose of the patient's long-acting anticonvulsant or waiting for anticonvulsant serum concentration results. It was also noted that the 23 patients diagnosed with febrile seizures tended to respond to treatment and few required rescue medication, intubation, hospitalization, or ICU care.

There are a number of limitations to consider when interpreting these study results. This was a secondary unplanned analysis and was not powered to demonstrate noninferiority for the given outcome in the pediatric subgroup of the RAMPART study population. Only descriptive data and estimates of differences and precision of the estimates are provided. Due to the nature of the study and analysis, no formal, experimentally-derived recommendations and conclusions to the safety and efficacy can be made. Many of the estimates of patient characteristics, times to events, safety profiles, and outcome measures were, however, similar to and consistent with the overall RAMPART study population. The findings within the pediatric subgroup are reassuring despite the preceding limitations. The emergent nature of the study and the fact that it was carried out in the prehos- 
pital setting dictated that estimated weights were used and that children were stratified to one of only two dose tiers. Some may worry that these broad fixed-dose tiers may result in doses too high for those at the lower end or too low for those at the higher of each stratum. We chose this fixeddose protocol to avoid the known problems with the more extreme dosing errors resulting from miscalculation and measurement error commonly encountered in pediatric resuscitation efforts. ${ }^{16}$ The range of doses in $\mathrm{mg} / \mathrm{kg}$ found at the extremes of each dose tier were consistent with the wide ranges reported in clinical use. Our study did not find any safety issues using this dose schedule but the possibility of narrower dose tiers could be the subject of future study. Although a limitation, the study design more accurately reflected the realities of the emergency prehospital treatment of this disorder. In the absence of such data, a fixedtier dosing remains more pragmatic, less susceptible to errors in administration, and consistent with a favorable safety profile.

In summary, this analysis of the RAMPART pediatric cohort reports the demographics and clinical characteristics of children treated by EMS for SE in the prehospital setting. Those treated tended to be preschool and young school-aged children, most of whom had a history of prior seizures. Noncompliance with medications is a less common cause of SE than in the overall cohort, but psychogenic nonepileptic spells are similar in frequency. Although there is insufficient power to independently assess efficacy and safety outcomes by intervention in the pediatric subgroup, the findings are consistent with those of the overall cohort and support the representativeness of the original analysis. Rates of hospital and ICU admission, endotracheal intubation, and recurrent seizure are all favorable in children compared to the overall cohort, and in IM midazolam compared with IV lorazepam.

\section{ACKnowledgments}

Grant Support: National Institute of Neurological Disorders and Stroke (NINDS) (U01NS056975 and U01NS059041); National Institutes of Health Office of the Director CounterACT Program; Biomedical Advanced Research and Development Authority of the Assistant Secretary for Preparedness and Response.

\section{Disclosure}

None of the authors has any conflict of interest to disclose. We confirm that we have read the Journal's position on issues involved in ethical publication and affirm that this report is consistent with those guidelines.

\section{Additional Contributors}

RDW, DL, RC, and RS conceived this study. RS and DL obtained funding for the original RAMPART study. RDW and RS designed the study. VLD and KN provided data management, statistical advice on the design, and analyzed the data. RDW drafted the manuscript and all authors contributed substantially to its revisions. RDW takes responsibility for the manuscript as a whole.

\section{REFERENCES}

1. Rossetti AO, Lowenstein DH. Management of refractory status epilepticus in adults: still more questions than answers. Lancet Neurol 2011;10:922-930.

2. Brophy GM, Bell R, Claassen J, et al. Guidelines for the evaluation and management of status epilepticus. Neurocrit Care 2012;17:3-23.

3. Alldredge BK, Gelb AM, Isaacs SM, et al. A comparison of lorazepam, diazepam, and placebo for the treatment of out-of-hospital status epilepticus. N Engl J Med 2001;345:631-637.

4. Towne AR, DeLorenzo RJ. Use of intramuscular midazolam for status epilepticus. J Emerg Med 1999;17:323-328.

5. Chamberlain JM, Altieri MA, Futterman C, et al. A prospective, randomized study comparing intramuscular midazolam with intravenous diazepam for the treatment of seizures in children. Pediatr Emerg Care 1997;13:92-94.

6. Silbergleit R, Durkalski V, Lowenstein D, et al. Intramuscular versus intravenous therapy for prehospital status epilepticus. $N$ Engl J Med 2012:366:591-600.

7. Silbergleit R, Lowenstein D, Durkalski V, et al. RAMPART (Rapid Anticonvulsant Medication Prior to Arrival Trial): a double-blind randomized clinical trial of the efficacy of intramuscular midazolam versus intravenous lorazepam in the prehospital treatment of status epilepticus by paramedics. Epilepsia 2011;52(Suppl. 8):45-47.

8. Hashikawa A, Juhn Y, Homme J, et al. Does length-based resuscitation tape accurately place pediatric patients into appropriate color-coded zones? Pediatr Emerg Care 2007;23:856-861.

9. RAMPART Protocol http://www.nejm.org/doi/suppl/10.1056/NEJM oa1107494/suppl_file/nejmoa1107494_protocol.pdf. N Engl J Med. 2012;366: See Table 5; Accessed October 20, 2014.

10. Lowenstein DH. Status epilepticus: an overview of the clinical problem. Epilepsia 1999;40(Suppl. 1):S3-S8; discussion S21-22.

11. Riviello JJ Jr, Claassen J, LaRoche SM, et al. Treatment of status epilepticus: an international survey of experts. Neurocrit Care 2013;18:193-200.

12. Chamberlain JM, Okada P, Holsti M, et al. Lorazepam vs diazepam for pediatric status epilepticus: a randomized clinical trial. JAMA 2014;311:1652-1660

13. McMullan J, Sasson C, Pancioli A, et al. Midazolam versus diazepam for the treatment of status epilepticus in children and young adults: a meta-analysis. Acad Emerg Med 2010;17:575-582.

14. de Haan GJ, van der Geest P, Doelman G, et al. A comparison of midazolam nasal spray and diazepam rectal solution for the residential treatment of seizure exacerbations. Epilepsia 2010;51:478-482.

15. Lahat E, Goldman M, Barr J, et al. Comparison of intranasal midazolam with intravenous diazepam for treating febrile seizures in children: prospective randomised study. BMJ 2000;321:83-86.

16. Kozer E, Seto W, Verjee Z, et al. Prospective observational study on the incidence of medication errors during simulated resuscitation in a paediatric emergency department. BMJ 2004;329:1321.

\section{APPENDix I \\ NeURological EMERGENCIES Treatment Trial (NETT) INVESTIGATORS FOR RAMPART}

Clinical Coordinating Center: William G. Barsan, Daniel Lowenstein, Lewis Morgenstern, Arthur Pancioli, Robert Silbergleit, Valerie Stevenson, Deneil Harney, Donna Harsh, Kay Vonderschmidt, Erin Zaleski.

Statistical Data Management Center: Yuko Palesch, Valerie Durkalski, Catherine Dillon.

National Institutes of Health: Robin Conwit, Scott Janis.

Hub Investigators and Coordinators: David Wright, Gerald Beltran, Andrea McDougal, Matthew Bitner, Harriet Howlett-Smith, Rachel Barnhard (Emory University). 


\section{R. D. Welch et al.}

Chris Lewandowski, Taher Vohra, Anna Baker, Deen Creech, Gregory Flynn, Paula Crouse (Henry Ford Health System).

Tom Aufderheide, Joseph Brandt, Riccardo Colella, Joanna Delap, Michel Torbey, Jennifer Noldin, Erin Brandenburg (Medical College of Wiscon$\sin )$.

Stephan Mayer, Neal Flomenbaum, Cristina Falo, Chirag Surti, Heidi Cordi (New York Presbyterian Hospital).

Robert Lowe, Craig Warden, Rachel Stone (Oregon Health and Science University).

James Quinn, Stephanie Casal, Peter Dsouza, Matt Hall (Stanford University).

Nina Gentile, Brent Freeman, Stacey Cleary, Christopher Vates, Alvin Wang (Temple University).

Kurt Denninghoff, Daniel Spaite, Bruce Barnhart, Willie Haro (University of Arizona).

Claude Hemphill, Michele Meeker, Jeany Duncan, Karl Sporer (University of California at San Francisco).
Art Pancioli, Hamilton Schwartz, Irene Ewing, Kay Vonderschmidt, Jason McMullan, Erin Grise (University of Cincinnati).

Roger Humphries, Linda Dechtenberg, Christofer Sweat, Robert Hendricks (University of Kentucky).

Barney Stern, Tricia Ting, Greg Krauss, Virginia Ganley, Susan Rice, Michelle Stevens, Greg Valcourt (University of Maryland).

Michelle Biros, Corey Sargent, Kathleen Miller (University of Minnesota).

Jill Baren, R. Daniel Bledsoe, Katie Lamond, Barbie Stahlman (University of Pennsylvania).

Elizabeth Jones, TJ Milling, Misty Ottman, Ben King, Louis Gonzales, Jeffrey Brockman, Gonnie Richter, David Anderson (University of Texas Houston).

Joseph Ornato, Sallie Noe, Alan Payne (Virginia Commonwealth University).

Robert Welch, LynnMarie Mango, Jenny Atas (Wayne State University). 